\title{
KEPLER-10 c: A 2.2 EARTH RADIUS TRANSITING PLANET IN A MULTIPLE SYSTEM
}

\author{
François Fressin $^{1}$, Guillermo Torres ${ }^{1}$, Jean-Michel Désert ${ }^{1}$, David Charbonneau ${ }^{1}$, Natalie M. Batalha ${ }^{2}$, \\ Jonathan J. Fortney ${ }^{3}$, Jason F. Rowe ${ }^{4,17}$, Christopher Allen, ${ }^{4,5}$, William J. BoruCKi ${ }^{4}$, Timothy M. Brown ${ }^{6}$, \\ Stephen T. Bryson ${ }^{4}$, David R. Ciardi ${ }^{7}$, Willliam D. Cochran ${ }^{8}$, Drake Deming $^{9}$, Edward W. Dunham ${ }^{10}$, \\ Daniel C. Fabrycky ${ }^{3}$, Thomas N. Gautier III ${ }^{11}$, Ronald L. Gilliland ${ }^{12}$, Christopher E. Henze ${ }^{4}$, Matthew J. Holman ${ }^{1}$, \\ Steve B. Howell ${ }^{13}$, Jon M. Jenkins ${ }^{4,14}$, Karen Kinemuchi ${ }^{4,15}$, Heather Knutson ${ }^{16}$, David G. Koch $^{7}$, \\ David W. Latham ${ }^{1}$, Jack J. Lissauer ${ }^{4}$, Geoffrey W. Marcy ${ }^{16}$, Darin Ragozzine ${ }^{1}$, \\ Dimitar D. Sasselov ${ }^{1}$, Martin Still ${ }^{4,15}$, Peter Tenenbaum ${ }^{4,5}$, and Kamal Uddin ${ }^{4,5}$ \\ ${ }^{1}$ Harvard-Smithsonian Center for Astrophysics, Cambridge, MA 02138, USA; ffressin@cfa.harvard.edu \\ ${ }^{2}$ Department of Physics, San Jose State University, San Jose, CA 95192, USA \\ ${ }^{3}$ Department of Astronomy and Astrophysics, University of California, Santa Cruz, CA 95064, USA \\ ${ }^{4}$ NASA Ames Research Center, Moffett Field, CA 94035, USA \\ ${ }^{5}$ Uddin Orbital Sciences Corporation, Moffett Field, CA 94035, USA \\ ${ }^{6}$ Las Cumbres Observatory Global Telescope, Goleta, CA 93117, USA \\ ${ }^{7}$ NASA Exoplanet Science Institute/Caltech, Pasadena, CA 91125, USA \\ ${ }^{8}$ McDonald Observatory, University of Texas at Austin, Austin, TX 78712, USA \\ ${ }^{9}$ NASA Goddard Space Flight Center, Greenbelt, MD 20771, USA \\ ${ }^{10}$ Lowell Observatory, Flagstaff, AZ 86001, USA \\ ${ }^{11}$ Jet Propulsion Laboratory/California Institute of Technology, Pasadena, CA 91109, USA \\ ${ }^{12}$ Space Telescope Science Institute, Baltimore, MD 21218, USA \\ ${ }^{13}$ National Optical Astronomy Observatory, Tucson, AZ 85719, USA \\ ${ }^{14}$ SETI Institute/NASA Ames Research Center, Moffett Field, CA 94035, USA \\ ${ }_{15}$ Bay Area Environmental Research Institute, Sonoma, CA 95476, USA \\ ${ }^{16}$ Astronomy Department, University of California, Berkeley, CA 94720, USA \\ Received 2011 April 9; accepted 2011 April 30; published 2011 October 10
}

\begin{abstract}
The Kepler mission has recently announced the discovery of Kepler-10 b, the smallest exoplanet discovered to date and the first rocky planet found by the spacecraft. A second, 45 day period transit-like signal present in the photometry from the first eight months of data could not be confirmed as being caused by a planet at the time of that announcement. Here we apply the light curve modeling technique known as BLENDER to explore the possibility that the signal might be due to an astrophysical false positive (blend). To aid in this analysis we report the observation of two transits with the Spitzer Space Telescope at $4.5 \mu \mathrm{m}$. When combined, they yield a transit depth of $344 \pm 85 \mathrm{ppm}$ that is consistent with the depth in the Kepler passband ( $376 \pm 9$ ppm, ignoring limb darkening), which rules out blends with an eclipsing binary of a significantly different color than the target. Using these observations along with other constraints from high-resolution imaging and spectroscopy, we are able to exclude the vast majority of possible false positives. We assess the likelihood of the remaining blends, and arrive conservatively at a false alarm rate of $1.6 \times 10^{-5}$ that is small enough to validate the candidate as a planet (designated Kepler-10 c) with a very high level of confidence. The radius of this object is measured to be $R_{p}=2.227_{-0.057}^{+0.052} R_{\oplus}$ (in which the error includes the uncertainty in the stellar properties), but currently available radial-velocity measurements only place an upper limit on its mass of about $20 M_{\oplus}$. Kepler-10 c represents another example (with Kepler-9 d and Kepler-11 g) of statistical "validation" of a transiting exoplanet, as opposed to the usual "confirmation" that can take place when the Doppler signal is detected or transit timing variations are measured. It is anticipated that many of Kepler's smaller candidates will receive a similar treatment since dynamical confirmation may be difficult or impractical with the sensitivity of current instrumentation.
\end{abstract}

Key words: binaries: eclipsing - planetary systems - stars: individual (Kepler-10, KOI-072, KIC 11904151) stars: statistics

Online-only material: color figures

\section{INTRODUCTION}

The Kepler mission has recently made public a catalog of all transiting planet candidates identified during the first four months of observation by the spacecraft (Borucki et al. 2011b). Included in this list of 1235 objects are nearly 300 in the category of super-Earths (defined here as having radii in the range $1.25 R_{\oplus}<R_{p}<2 R_{\oplus}$ ), and several dozen of Earth-size $\left(R_{p}<1.25 R_{\oplus}\right)$. The wealth of new information

\footnotetext{
${ }^{17}$ NASA Postdoctoral Program Fellow.
}

promises to revolutionize our knowledge of extrasolar planets. Although strictly speaking these are still only candidates since confirmation by spectroscopic or other means is not yet in hand, expectations are high that the rate of false positives in this list is relatively small (see Borucki et al. 2011b; Morton \& Johnson 2011). Consequently, results from this sample concerning the general properties of exoplanets have already begun to emerge, including studies of the architecture and dynamics of multiple transiting systems (Lissauer et al. 2011b), an investigation of the statistical distribution of eccentricities (Moorhead et al. 2011), and first estimates of the rate of occurrence of planets larger 
than $2 R_{\oplus}$ with orbital periods up to 50 days (A. W. Howard et al. 2011, in preparation), among others.

For good reasons the confirmation or "validation" of small transiting planets ${ }^{18}$ (Earth-size or super-Earth-size) has attracted considerable attention, but has proven to be non-trivial in many cases because of the difficulty of detecting the tiny radialvelocity (RV) signatures that these objects cause on their parent stars, as exemplified by the cases of CoRoT-7 b (Léger et al. 2009), Kepler-9 d (Torres et al. 2011), and Kepler-11 g (Lissauer et al. 2011a). In fact, such spectroscopic signals are often too small to detect with current instrumentation, and the planetary nature of the candidate must be established statistically, as in the latter two cases.

The smallest planet discovered to date, Kepler-10 b, was announced recently by Batalha et al. (2011), and is the Kepler mission's first rocky planet. It has a measured radius of $1.416_{-0.036}^{+0.033} R_{\oplus}$ and a mass of $4.6_{-1.3}^{+1.2} M_{\oplus}$, leading to a mean density of $8.8_{-2.9}^{+2.1} \mathrm{~g} \mathrm{~cm}^{-3}$ which implies a significant iron mass fraction (Batalha et al. 2011). Its parent star, Kepler-10 (KIC 11904151, 2MASS 119024305+5014286), is relatively bright among the Kepler targets (Kepler magnitude $K p=10.96)$ and displays two periodic signals with periods of 0.84 days and 45.3 days, and flux decrements (ignoring limb darkening) of $152 \pm 4$ ppm and $376 \pm 9$ ppm, respectively (Batalha et al. 2011). The extensive observations that followed the detection of these signals are documented in detail by those authors, and include the difficult measurement of the reflex RV motion of the star with a semi-amplitude of only $3.3_{-1.0}^{+0.8} \mathrm{~m} \mathrm{~s}^{-1}$ and a period that is consistent with the shorter signal. As is customary also in ground-based searches for transiting planets, the shapes of the spectral lines were examined carefully to rule out changes of similar amplitude correlating with orbital phase that might indicate a false positive, such as a background eclipsing binary (EB) blended with the target, or an EB physically associated with it. However, the precision of the measurements (bisector spans) compared to the small RV amplitude did not allow such changes to be ruled out unambiguously. False positive scenarios were explored with the aid of BLENDER, a technique that models the transit light curves to test a wide range of blend configurations (Torres et al. 2011), and it was found that the overwhelming majority of them can be rejected. This and other evidence presented by Batalha et al. (2011) allowed the planetary nature of Kepler-10 b to be established with very high confidence.

This was not the case, however, for the 45 day period signal referred to as KOI-072.02 (Kepler Object of Interest 72.02), which is the subject of this paper. No significant RV signal was detected at this period, and only an upper limit on its amplitude could be placed. Using BLENDER, Batalha et al. (2011) were able to rule out a large fraction of the blend scenarios involving circular orbits (including hierarchical triples), but eccentric orbits were not explored because of the increased complexity of the problem and the much larger space of parameters for false positives. While circular orbits are a reasonable assumption for Kepler-10 b because of the strong effects of tidal forces at close range, this is not true for KOI-072.02 on account of its much longer orbital period (see, e.g., Mazeh 2008); eccentric orbits cannot be ruled out.

\footnotetext{
18 In the context of this paper "confirmation" refers to the unambiguous detection of the gravitational influence of the planet on its host star or on other bodies in the system (e.g., the Doppler signal or transit timing variations) to establish the planetary nature of the candidate; when this is not possible, we speak of "validation," which involves an estimate of the false alarm probability.
}

This provides the motivation for the present work, in which we set out to examine all viable astrophysical false positive scenarios for KOI-072.02 with the goal of validating it as a bona fide planet. In addition to improvements in the BLENDER modeling, we bring to bear new near-infrared observations obtained with the Spitzer Space Telescope in which the transits are clearly detected as well as the complete arsenal of follow-up observations gathered by the Kepler team, including high-resolution adaptive optics (AO) imaging and speckle interferometry, highresolution spectroscopy, and an analysis based on the Kepler observations themselves of the difference images in and out of transit for positional displacements (centroid motion). All of these observations combined with the strong constraints provided by BLENDER significantly limit the kinds of blends that remain possible, and as we describe below they allow us to claim with very high confidence that KOI-072.02 is indeed a planet. Its estimated radius is approximately $60 \%$ of that of Neptune. With this, Kepler-10 becomes the mission's third confirmed multi-planet system (after Kepler-9 and Kepler-11; Holman et al. 2010; Lissauer et al. 2011a) containing a transiting super-Earth-size planet and at least one larger planet that also transits.

We begin with a brief recapitulation of the BLENDER technique, including recent improvements. We then present the Warm Spitzer observations at $4.5 \mu \mathrm{m}$ that help rule out many blends, and we summarize additional constraints available from other observations. This is followed by the application of BLENDER to KOI-072.02 in order to identify all blend scenarios that can mimic the Kepler transit light curve. Next we combine this information with the other constraints and carry out a statistical assessment of the false alarm rate (FAR) for the planet hypothesis, leading to the validation of the candidate as Kepler-10 c. We conclude with a discussion of the possible constitution of the new planet in the light of current models, and the significance of this type of validation.

\section{REJECTING FALSE POSITIVES WITH BLENDER}

The detailed morphology of a transit light curve (length of ingress/egress, total duration) contains important information that can be used to reject many false positive scenarios, producing brightness variations that do not quite have the right shape even though they may well match the observed transit depth (see, e.g., Snellen et al. 2009). BLENDER (Torres et al. 2004, 2011) takes advantage of this to explore a very large range of scenarios, including background or foreground eclipsing binaries blended with the target as well as eclipsing binaries physically associated with the target in a hierarchical triple configuration. Following the notation introduced by Torres et al. (2011), the objects composing the binary are referred to as the "secondary" and "tertiary," and the candidate is the "primary." The tertiary can be either a star (including a white dwarf) or a planet, and the secondary can be a main-sequence star or a (background) giant.

With the help of model isochrones to set the stellar properties, BLENDER simulates blend light curves resulting from the flux of the eclipsing pair diluted by the brighter target (and any additional stars that may fall within the photometric aperture). Each simulated light curve is compared with the Kepler observations in a $\chi^{2}$ sense to identify which of them result in acceptable fits (to be defined later). The parameters varied during the simulations are the mass of the secondary star $\left(M_{2}\right)$, the mass of the tertiary ( $M_{3}$, or its radius $R_{3}$ if a planet), the impact parameter $(b)$, the relative linear distance $(d)$ between the eclipsing pair and the target, and the relative duration $\left(D / D_{\text {circ }}\right)$ of the transit compared 
to the duration for a circular orbit (see below). For convenience, the relative linear distance is parameterized in terms of the difference in distance modulus, $\Delta \delta$, where $\Delta \delta=5 \log \left(d_{\mathrm{EB}} / d_{\mathrm{KOI}}\right)$. In the case of hierarchical triple configurations, the isochrone for the binary is assumed to be the same as for the primary (metallicity of $[\mathrm{Fe} / \mathrm{H}]=-0.15$ and a nominal age of $11.9 \mathrm{Gyr}$; see Batalha et al. 2011), whereas for background blends we have adopted for the binary a representative $3 \mathrm{Gyr}$ isochrone of solar metallicity although these parameters have a minimal impact on the results. For full details of the technique we refer the reader to the references above. Three recent changes and improvements that are especially relevant to the application to KOI-072.02 are described next.

1. The relatively long orbital period of KOI-072.02 (45.3 days) precludes us from assuming that the eccentricity $(e)$ is zero, as we were able to suppose in previous applications of BLENDER to Kepler-9 d and Kepler-10 b, which have periods of 1.59 and 0.84 days, respectively. The reason that matters is that the duration of the transit is set, among other factors, by the size of the secondary star. Eccentricity can alter the speed of the tertiary around the secondary, making it slower or faster than in the circular case depending on the orientation of the orbit (longitude of periastron, $\omega$ ). Given a fixed (measured) duration, blends with smaller or larger secondary stars than in the circular case may still provide satisfactory fits to the light curve, effectively increasing the pool of potential false positives.

BLENDER now takes this into account, although rather than using $e$ and $\omega$, which are the natural variables employed in the binary light curve generating routine at the core of BLENDER (see Torres et al. 2011) as parameters, a more convenient variable that captures the effects of both is the duration relative to a circular orbit. Following Winn (2010), this may be expressed as $D / D_{\text {circ }} \approx \sqrt{1-e^{2}} /(1+e \sin \omega)$. Operationally, then, we vary $D / D_{\text {circ }}$ over wide ranges as we explore different blend scenarios, and for each value, we infer the corresponding values of $e$ and $\omega$. In practice, in order to solve for $\{e, \omega\}$ from $D / D_{\text {circ }}$ it is only necessary to consider the limiting cases with $\omega=90^{\circ}$ and $270^{\circ}$, corresponding to transits occurring at periastron and apastron, respectively, since these are the orientations resulting in the minimum and maximum durations for a given eccentricity. Other combinations of $e$ and $\omega$ will lead to intermediate relative durations that are already sampled in our $D / D_{\text {circ }}$ grid. It is worth noting that use of only these two values of $\omega$ leads to predicted secondary eclipses in the simulated light curves that are always located at phase 0.5 , whereas secondary eclipses in the real data might be present at any phase. For our purposes, this is of no consequence, as KOI-072.02 has already had its light curve screened for secondary eclipses at any phase that might betray a false positive as part of the vetting process. No such features are present down to the $100 \mathrm{ppm}$ level. Thus, any simulated light curves from BLENDER that display a significant secondary eclipse will yield poor fits no matter where the secondary eclipse happens to be, and will lead to the rejection of that particular blend scenario.

2. For each false positive configuration BLENDER can predict the overall photometric color of the blend, for comparison with the measured color index of the candidate as reported in the Kepler Input Catalog (KIC; Brown et al. 2011). A color index such as $K p-K_{s}$, where $K p$ is the Kepler magnitude and $K_{s}$ is derived from the Two Micron All
Sky Survey catalog, provides a reasonable compromise between wavelength leverage and the precision of the index. The latter varies typically between 0.015 and 0.030 mag depending on the passband and the brightness of the star (see Brown et al. 2011). We consider a particular blend to be rejected when its predicted color deviates from the KIC value by more than three times the error of the latter. As it turns out, color is a particularly effective way of rejecting blends that include secondary stars of a different spectral type than the primary, such as those that become possible when allowing for eccentric orbits.

3. Recent refinements in the resolution of the BLENDER simulations to better explore parameter space, in addition to the inclusion of eccentricity (or $D / D_{\text {circ }}$ ) as an extra variable, have increased the complexity of the problem as well as the computing time (by nearly two orders of magnitude) compared to the relatively simple case of circular orbits. The number of different parameter combinations examined with BLENDER (and corresponding light curve fits) can approach $7 \times 10^{8}$ in some cases. Consequently, the simulations are now performed on the Pleiades cluster at the NASA Advanced Supercomputing Division, located at the Ames Research Center (California), typically on 1024 processors running in parallel. For convenience hierarchical triple configurations (four parameters) and background/foreground blends (five parameters) are studied separately, each for the two separate cases of stellar and planetary tertiaries (for a total of four grids). One additional fit is carried out using a true transiting planet model to provide a reference for the quality of the false positive fits in the other grids.

The discriminating value of the shape information contained in the light curves, mentioned at the beginning of this section, is highlighted by our BLENDER results for Kepler-10 b, as described by Batalha et al. (2011). In that study it was found that all background EB configurations with stellar tertiaries yield very poor fits to the Kepler light curve and are easily rejected. The underlying reason is that all such blend models predict obvious brightness changes out of eclipse (ellipsoidal variations) with an amplitude that is not seen in the data, and which are a consequence of the very short orbital period. ${ }^{19}$ Hierarchical triple scenarios were also excluded based on joint constraints from BLENDER and other follow-up observations. The only configurations providing suitable alternatives to the true planet scenario involved stars in the foreground or background of the target that are orbited by a larger transiting planet. The considerable reduction in the blend frequency (BF) from the exclusion of all background eclipsing binaries led to a false alarm probability low enough to validate Kepler-10 b with a very high level of confidence, independently of any spectroscopic evidence. This remarkable result speaks to the power of BLENDER when combined with all other observational constraints. It also assumes considerable significance for Kepler-10 b, given that it was not

\footnotetext{
19 Note that the present post-processing of Kepler data in preparation for the BLENDER analyses (see Section 4) artificially suppresses out-of-eclipse

variations to some extent, typically by median filtering, so that the light curves for periods as short as that of Kepler-10 b (0.84 days) are rendered essentially flat except for the transits themselves. In this sense, the situation is similar to that mentioned earlier regarding the presence of secondary eclipses: obvious ellipsoidal variability in the raw data would normally trigger a false positive warning during the vetting process, preventing the target from becoming an object of interest. But if it reaches KOI status, we assume that out-of-eclipse modulations are insignificant so that the comparison with any BLENDER model in which those variations are present is meaningful and would yield a poor fit, sufficient in most cases to reject the blend.
} 
possible to provide separate proof of the planetary nature of this signal in the Batalha et al. (2011) study from an examination of the bisector spans. The scatter of the bisector span measurements $\left(10.5 \mathrm{~m} \mathrm{~s}^{-1}\right)$ was three times larger than the RV semi-amplitude $\left(3.3 \mathrm{~m} \mathrm{~s}^{-1}\right)$, rendering them inconclusive.

The situation regarding the BLENDER analysis of the KOI072.02 signal in the Batalha et al. (2011) study was very different: the orbital period is much longer, and ellipsoidal variations are predicted to be negligible, so that background eclipsing binaries with stellar tertiaries remain viable blends. This, and the added complication from eccentric orbits, hindered the efforts of those authors to validate this candidate. With the benefit of the enhancements in BLENDER described above, we are now in a better position to approach this problem anew.

As follow-up observations provide important constraints that are complementary to those supplied by BLENDER, and play an important role in determining the FAR for the planetary nature of KOI-072.02 (Section 6), we describe those first below, beginning with our new near-infrared Spitzer observations.

\section{OBSERVATIONAL CONSTRAINTS}

\subsection{Warm Spitzer Observations of KOI-072.02}

KOI-072.02 was observed during two transits with the IRAC instrument on the Spitzer Space Telescope (Werner et al. 2004; Fazio et al. 2004) at $4.5 \mu \mathrm{m}$ (program ID 60028). The observations were obtained on UT 2010 August 30 and November 15, with each visit lasting approximately $15 \mathrm{hr}$ 10 minutes. The data were gathered in full-frame mode $(256 \times$ 256 pixels) with an exposure time of $6.0 \mathrm{~s}$ per image, which resulted in approximately a $7.1 \mathrm{~s}$ cadence and yielded 7700 images per visit.

The method we used to produce photometric time series from the images is described by Désert et al. (2009). It consists of finding the centroid position of the stellar point-spread function (PSF) and performing aperture photometry using a circular aperture on individual exposures. The images used are the Basic Calibrated Data delivered by the Spitzer archive. These files are corrected for dark current, flat fielding, and detector nonlinearity, and are converted to flux units. We converted the pixel intensities to electrons using the information on the detector gain and exposure time provided in the FITS headers. This facilitates the evaluation of the photometric errors. We extracted the UTC-based Julian date for each image from the FITS header (keyword DATE_OBS) and corrected to midexposure. We converted to TDB-based barycentric Julian dates using the UTC2BJD ${ }^{20}$ procedure developed by Eastman et al. (2010). This program uses the JPL Horizons ephemeris to estimate the position of the Spitzer spacecraft during the observations. We then corrected for transient pixels in each individual image using a 20 point sliding median filter of the pixel intensity versus time. To do so, we compared each pixel's intensity to the median of the 10 preceding and 10 following exposures at the same pixel position, and we replaced outliers greater than $4 \sigma$ with their median value. The fraction of all pixels we corrected is $0.02 \%$ for the first visit and $0.06 \%$ for the second.

The centroid position of the stellar PSF was determined using the DAOPHOT-related procedures GCNTRD, from the IDL Astronomy Library. ${ }^{21}$ We applied the APER routine to perform

\footnotetext{
20 http://astroutils.astronomy.ohio-state.edu/time/

$21 \mathrm{http} / / /$ idlastro.gsfc.nasa.gov/homepage.html
}

aperture photometry with a circular aperture of variable radius, using a range of radii between 1.5 and 8 pixels in steps of 0.5 . The propagated uncertainties were derived as a function of the aperture radius, and we adopted the aperture providing the smallest errors. We found that the transit depths and errors varied only weakly with aperture radius for all light curves analyzed in this project. The optimal aperture was found to have a radius of 4.0 pixels.

We estimated the background by examining a histogram of counts from the full array. We fit a Gaussian curve to the central region of this distribution (ignoring bins with high counts, which correspond to pixels containing stars), and we adopted the center of this Gaussian as the value of the residual background intensity. As seen already in previous Warm Spitzer observations (Deming et al. 2011; Beerer et al. 2011), we found that the background varies by $20 \%$ between three distinct levels from image to image, and displays a ramp-like behavior as function of time. The contribution of the background to the total flux from the stars is low for both observations, from $0.1 \%$ to $0.55 \%$ depending on the image. Therefore, photometric errors are not dominated by fluctuations in the background. We used a sliding median filter to select and trim outliers in flux and position greater than $5 \sigma$, representing $1.6 \%$ and $1.3 \%$ of the data for the first and second visits, respectively. We also discarded the first half-hour's worth of observations, which is affected by significant telescope jitter before stabilization. The final number of photometric measurements used is 7277 and 7362 .

The raw time series are presented in the top panel of Figure 1. We find that the point-to-point scatter in the photometry gives a typical signal-to-noise ratio $(\mathrm{S} / \mathrm{N})$ of 280 per image, which corresponds to $90 \%$ of the theoretical $\mathrm{S} / \mathrm{N}$. Therefore, the noise is dominated by Poisson statistics.

\subsection{Analysis of the Warm Spitzer Light Curves and Results}

In order to determine the transit parameters and associated uncertainties from the Spitzer time series we used a transit light curve model multiplied by instrumental decorrelation functions, as described by Désert et al. (2011a). The transit light curves were computed with the IDL transit routine OCCULTSMALL from Mandel \& Agol (2002). For the present case we allowed for a single free parameter in the model, which is the planet-to-star radius ratio $R_{p} / R_{\star}$ (or equivalently, the depth, in the absence of limb darkening). The normalized orbital semimajor axis (system scale) $a / R_{\star}$, the impact parameter $b$, the period $P$, and the time of mid-transit $T_{c}$ were held fixed at the values derived from the Kepler light curve, as reported by Batalha et al. (2011) and summarized below in Section 7. Limb darkening is small at $4.5 \mu \mathrm{m}$, but was nevertheless included in our modeling using the four-parameter law by Claret (2000) and theoretical coefficients published by Sing (2010).

The Spitzer/IRAC photometry is known to be systematically affected by the so-called pixel-phase effect (see, e.g., Charbonneau et al. 2005; Knutson et al. 2008). This effect is seen as oscillations in the measured fluxes with a period corresponding to that of the telescope pointing jitter. For the first visit this period was 70 minutes, and the amplitude of the oscillations was approximately $2 \%$ peak to peak; for the second visit the period was 35 minutes, and the amplitude about $1 \%$. We decorrelated our signal in each channel using a linear function of time for the baseline (two parameters) and a quadratic function of the PSF position (four parameters) to correct the data for each channel. We performed a simultaneous Levenberg-Marquardt least-squares fit to the data (Markwardt 2009) to determine the 


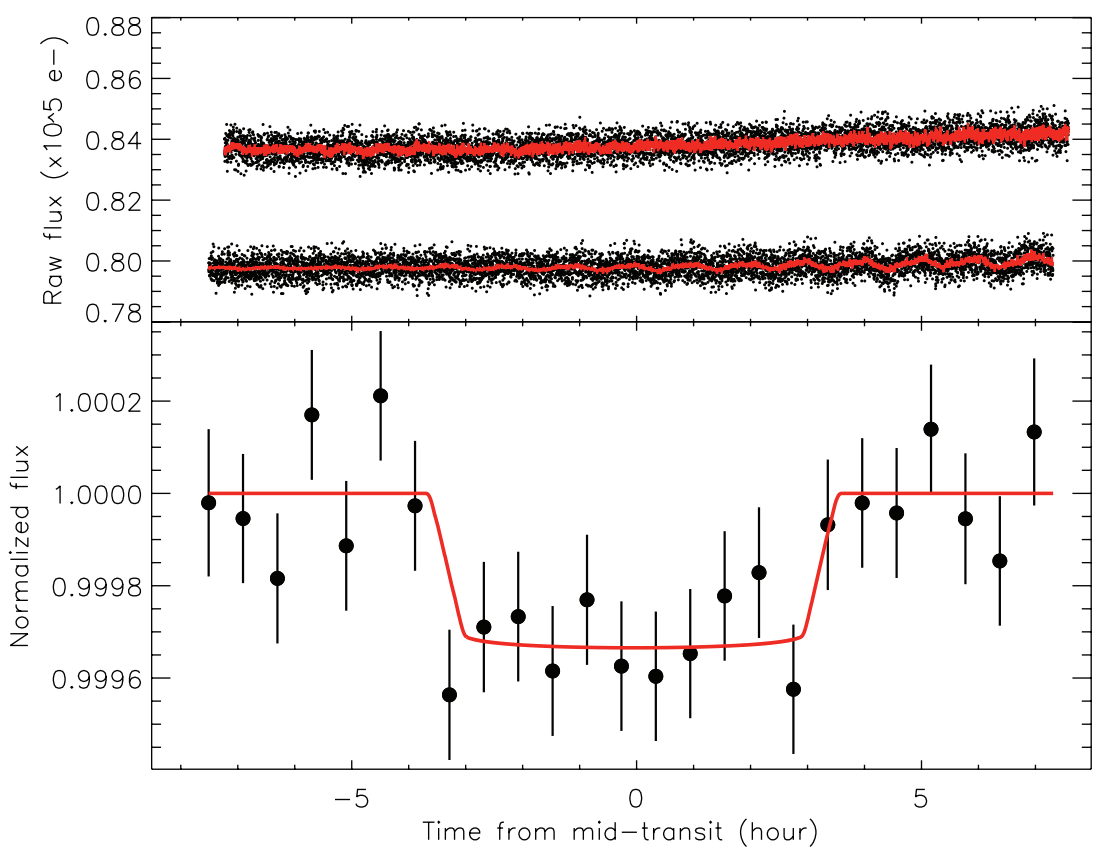

Figure 1. Spitzer transit light curves of KOI-072.02 observed in the IRAC bandpass at $4.5 \mu \mathrm{m}$. Top: raw measurements (black points) with the same data binned by two superimposed (12 s bins, red points). Bottom: measurements combined from the two visits and binned in 36 minute bins (295 points per bin), along with the best-fit limb-darkened transit model (integrated over the same duration). Both the data and the model shown here have been corrected for instrumental errors.

(A color version of this figure is available in the online journal.)

transit and instrumental model parameters (seven in total). The errors on each photometric point were assumed to be identical and were set to the rms residual of the initial best fit. To obtain an estimate of the correlated and systematic errors in our measurements (Pont et al. 2006) we used the residual permutation bootstrap technique, or "Prayer Bead" method, as described by Désert et al. (2009). In this method the residuals of the initial fit are shifted systematically and sequentially by one frame, and then added to the transit light curve model before fitting again. We considered asymmetric error bars spanning $34 \%$ of the points above and below the median of the distributions to derive the $1 \sigma$ uncertainties for each parameter, as described by Désert et al. (2011b).

The bottom panel of Figure 1 shows the best-fit model superimposed on the observations from the two visits combined, with the data binned in 36 minute bins for clarity (295 points per bin). The transit depths at $4.5 \mu \mathrm{m}$ (after removing limbdarkening effects) are $353_{-133}^{+115} \mathrm{ppm}$ for the first visit and $339_{-110}^{+85}$ for the second, which are in good agreement with each other. The weighted average depth of $344 \pm 85$ is consistent with the non-limb-darkened value of $376 \pm 9 \mathrm{ppm}$ derived from the Kepler light curve (Batalha et al. 2011) well within the $1 \sigma$ errors, strongly suggesting the transit is achromatic, as expected for a planet.

The above Spitzer observations provide a useful constraint on the kinds of false positives (blends) that may be mimicking the KOI-072.02 signal. For example, if Kepler-10 were blended with a faint unresolved background EB of much later spectral type that manages to reproduce the transit depth in the Kepler passband, the predicted depth at $4.5 \mu \mathrm{m}$ may be expected to be larger because of the higher flux of the contaminating binary at longer wavelengths compared to Kepler-10. Since the transit depth we measure in the near-infrared is about the same as in the optical, this argues against blends composed of stars of much later spectral type. Based on model isochrones and the properties of the target star (see below), we determine an upper limit to the secondary masses of $0.77 M_{\odot}$. This Spitzer constraint is used in Section 4 to eliminate many blends.

\subsection{Additional Observational Constraints on Possible False Positives}

Further constraints of a different kind are provided by highresolution imaging as described in more detail by Batalha et al. (2011). Briefly, these consist of speckle observations obtained on UT 2010 June 18 with a two-color (approximately $V$ and $R$ ) speckle camera on the WIYN 3.5 m telescope on Kitt Peak (see Howell et al. 2011), and near-infrared ( $J$-band) AO observations conducted on UT 2009 September 8 with the PHARO camera on the $5 \mathrm{~m}$ Palomar telescope. No companions were detected around Kepler-10 within 1".5 (for speckle) or 12'.5 (AO), and more generally these observations place strong limits on the presence of other stars as a function of angular separation (down to $0^{\prime \prime} .05$ in the case of speckle) and relative brightness (companions as faint as $\Delta J=9.5$ for $\mathrm{AO}$ ). These sensitivity curves are shown in Figure 9 of Batalha et al. (2011), and we make use of that information below.

High-resolution spectra described also by Batalha et al. (2011) and obtained with the HIRES instrument on the $10 \mathrm{~m}$ Keck I telescope place additional limits on the presence of close companions falling within the spectrograph slit $\left(0{ }^{\prime \prime} 87\right)$, such that stars within about 2 mag of the target would generally have been seen. A small chance remains that these companions could escape detection if their RV happens to be within a few $\mathrm{km} \mathrm{s}^{-1}$ of that of the target (which is a narrow-lined, slowly rotating star with $v \sin i=0.5 \pm 0.5 \mathrm{~km} \mathrm{~s}^{-1}$; Batalha et al. 2011), so that the spectral lines are completely blended. This would be extremely unlikely for a chance alignment with a background/foreground star, but not necessarily for physically associated companions in wide orbits, i.e., with slow orbital 
motions. We explored this through Monte Carlo simulations. The results indicate that the probability of having a physical companion within a conservative range of $\pm 10 \mathrm{~km} \mathrm{~s}^{-1}$ of the $\mathrm{RV}$ of the target that would also go unnoticed in our speckle observations, and that additionally would not induce a RV drift on the target large enough to have been detected in the highprecision measurements of Batalha et al. (2011), is only about $0.1 \%$.

Finally, an analysis of the image centroids measured from the Kepler observations rules out background objects of any brightness beyond about $2^{\prime \prime}$ of the target. This exclusion limit (equivalent to half a pixel) is considerably more conservative than the 0.6 reported by Batalha et al. (2011), and accounts for saturation effects not considered earlier (given that at $K p=$ 10.96 the star is very bright by Kepler standards) as well as quarter-to-quarter variations (where "quarters" usually represent three-month observing blocks interrupted by spacecraft rolls required to maintain the proper illumination of the solar panels).

\section{APPLICATION OF BLENDER TO KOI-072.02}

The Kepler photometry used here is the same as employed in the work of Batalha et al. (2011) and was collected between 2009 May 2 and 2010 January 9. These dates correspond to Kepler Quarter 0 (first nine days of commissioning data) through the first month of Quarter 4. For this study we used only the longcadence observations $(10,870$ measurements $)$ obtained by the spacecraft at regular intervals of about 29.4 minutes. All blend models generated with BLENDER were integrated over this time interval for comparison with the measurements. The original data have been de-trended for this work by removing a firstorder polynomial, and then applying median filtering with a two-day wide sliding window. Observations that occur during transits were masked and did not contribute to the median calculation. Because this sliding window is considerably shorter than the 45.3 day orbital period, any ellipsoidal variations present in the original data should be largely preserved, although in any case they are expected to be very small for binaries with periods as long as this. We adopted also the ephemeris of midtransit for KOI-072.02 as reported by Batalha et al. (2011), which is $T_{c}[\mathrm{BJD}]=2,454,971.6761+N \times 45.29485$ days, where $N$ is the number of cycles from the reference epoch.

Because it is relatively bright $(K p=10.96)$, Kepler-10 was also observed by the mission with a shorter cadence of approximately 1 minute for a period of several months to allow an asteroseismic characterization of the star. A total of 19 oscillation frequencies were detected, and enabled a very precise determination of the mean stellar density. When combined with stellar evolution models and a spectroscopic determination of the effective temperature and chemical composition, the resulting parameters for the star are very well determined. Kepler-10 is relatively old $(>7.4 \mathrm{Gyr})$ but is otherwise quite similar to the Sun, with a temperature of $T_{\text {eff }}=5627 \pm 44 \mathrm{~K}$, a mass and radius of $M_{\star}=0.895 \pm 0.060 M_{\odot}$ and $R_{\star}=$ $1.056 \pm 0.021 R_{\odot}$, and a composition $[\mathrm{Fe} / \mathrm{H}]=-0.15 \pm 0.06$ slightly below solar (Batalha et al. 2011).

As indicated earlier we considered four general scenarios for false positives: chance alignments (a pair of background/foreground eclipsing objects) and hierarchical triple systems, each with tertiaries that can be either stars or planets. The free parameters were varied over the following ranges: secondary mass $M_{2}$ between 0.10 and $1.40 M_{\odot}$, in steps of $0.02 M_{\odot}$; tertiary mass $M_{3}$ between 0.10 and $M_{2}$, also in steps of $0.02 M_{\odot}$; tertiary radius $R_{3}$ between 0.06 and $2.00 R_{\text {Jup }}$ in steps of $0.02 R_{\mathrm{Jup}}$; impact parameter $b$ between 0.00 and 1.00 in steps of 0.05 ; relative duration $D / D_{\text {circ }}$ between 0.2 and 4.6 in steps of 0.2 , corresponding to eccentricities up to 0.92 and values of $\omega$ of $90^{\circ}$ and $270^{\circ}$ (see Section 2); and relative distance $\Delta \delta$ (distance modulus difference) between -5.0 and +9.0 in steps of $0.5 \mathrm{mag}$, except for hierarchical triple configurations, for which $\Delta \delta=0$.

The goodness of the fit of each of the large number of synthetic light curves generated by BLENDER is quantified here by computing the $\chi^{2}$ statistic and comparing it with that of the best planet model fit. The difference can be assigned a significance level (or FAR) that depends on the number of free parameters of the problem. For example, for a blend scenario corresponding to a hierarchical triple system (4 degrees of freedom), a trial model giving a worse fit than the planet solution by $\Delta \chi^{2}=4.72$ is statistically different at the $1 \sigma$ level, assuming Gaussian errors (see, e.g., Press et al. 1992). A fit that is worse by $\Delta \chi^{2}=16.3$ is different at the $3 \sigma$ level. Hierarchical triple blends giving poorer fits than this are considered here to be ruled out by the Kepler photometry. For background/foreground scenarios ( 5 degrees of freedom) the $3 \sigma$ blend rejection level is $\Delta \chi^{2}=$ 18.2.

\subsection{BLENDER Results}

In this section, we describe the simulations carried out for the four general blend configurations mentioned above. Although the secondaries for the background scenarios can in principle also be evolved stars (giants), as opposed to main-sequence stars, we consistently found that the transit light curves generated by such systems give a very poor match to the observations because they do not have the right shape (the ingress/egress phases are too long). Therefore, we restricted our exploration of parameter space to main-sequence stars only.

An additional possibility for a false positive may stem from an error in the determination of the orbital period. If the true period were twice the nominal value, alternating transit events would correspond to primary and secondary eclipses, implicating a blended EB. The primary and secondary eclipses would often (but not always) be of different depth. As part of the vetting process for each candidate, the Kepler team examines the even-numbered and odd-numbered events to look for differences in depth that may indicate a false positive of this kind. As described by Batalha et al. (2011), no significant differences were found for KOI-072.02 beyond the $2 \sigma$ level, where $\sigma$ represents the uncertainty in the transit depth $(9 \mathrm{ppm})$. Nevertheless, as the possibility still exists that the components of the EB are identical, experiments were run with BLENDER to examine the transit shape produced by such scenarios, and it was found that the ingress and egress phases are always much too long compared to the observations, as expected for two equalsize stars eclipsing each other. Thus, these scenarios are easily ruled out as well.

\subsubsection{Background Eclipsing Binaries (Star+Star)}

The simulations with BLENDER indicate that few background blend scenarios with stellar tertiaries are able to mimic the transit features in the light curve at an acceptable level, and they all correspond to somewhat eccentric orbits. In Figure 2, we show the goodness of fit of these scenarios, with the small closed $3 \sigma$ contour representing the region of parameter space within which the fits are satisfactory, according to the criteria given above. Only blends with secondary masses $M_{2}$ larger than about 1.3 $M_{\odot}$ are allowed, and the EB can only be within a small 


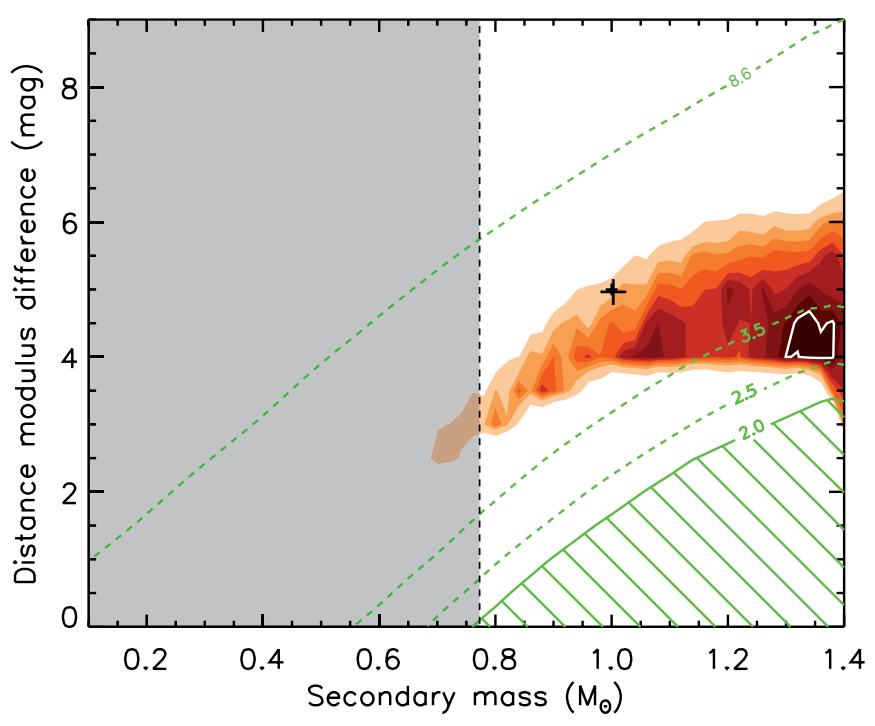

Figure 2. Map of the $\chi^{2}$ surface (goodness of fit) corresponding to a grid of blend models for KOI-072.02 involving background eclipsing binaries. The linear separation between the binary and the primary is cast in terms of the distance modulus difference. Contours are drawn as a function of the $\chi^{2}$ difference from the best planet model fit (expressed in units of the significance level of the difference, $\sigma$ ), and are plotted here as a function of the mass of the secondary star. Only blends within the small white contour yield acceptable fits to the light curve (within $3 \sigma$ of the planet fit). Other colored areas correspond to regions of parameter space giving increasingly worse fits $(4 \sigma, 5 \sigma$, etc.), representing blends we consider to be ruled out. The Spitzer constraint is indicated by the shaded area: blends with secondary masses in this region are excluded (see Section 3.2), although BLENDER itself already rules out all of these scenarios. Green lines running diagonally from the lower left to the upper right are labeled with the magnitude difference $\Delta K p$ of the blended binary relative to the target star. The hatched region below the $\Delta K p=2$ mag line represents blends with secondary stars bright enough that they would generally be detected in our spectroscopy. Viable blends within the $3 \sigma$ contour are seen to be confined to a narrow range of magnitude differences $(2.5 \leqslant \Delta K p \leqslant 3.5$, dashed green lines). The dashed line at $\Delta K p=8.6$ indicates the envelope for the faintest blends that would be capable of reproducing the measured depth based on simple-minded estimates described in the text. As seen, BLENDER provides much tighter constraints than this. The cross corresponds to a blend model that gives the fit shown in the bottom panel of Figure 3.

(A color version of this figure is available in the online journal.)

range of distances behind the target $(4.0 \lesssim \Delta \delta \lesssim 4.7)$ for the dilution effect to be just right, such that the corresponding apparent brightness difference $\Delta K p$ is between 2.5 and 3.5 mag (see figure). The best among these blend models (located near the center of the contour) provides a fit that is about $2.1 \sigma$ worse than a planet model (but still acceptable), and is shown in the top panel of Figure 3 compared against the planet model. The tertiary stars in these blends are constrained to be very small, between 0.10 and $0.16 M_{\odot}$.

That most blends involving background eclipsing binaries can be ruled out may appear somewhat surprising and is worth investigating. Indeed, for a given measured transit depth $d_{\text {tran }}$, a blend can only reproduce the light curve if it contributes at least a fraction $d_{\text {tran }}$ of the total flux collected in the Kepler aperture. Thus, one would expect that binaries as faint as $\Delta K p=-2.5 \log \left(d_{\text {tran }}\right) \approx 8.6$ mag relative to the target should be able to match that amount of dimming if they were totally eclipsed (see, e.g., Morton \& Johnson 2011), and furthermore, that the measured duration could also be reproduced by a large range of secondary sizes with an appropriate combination of orbital eccentricity and $\omega$. Yet we find that no blends fainter than $\Delta K p=3.5$ give tolerable fits to the light curve (see Figure 2). A visual understanding of the underlying reason for this may

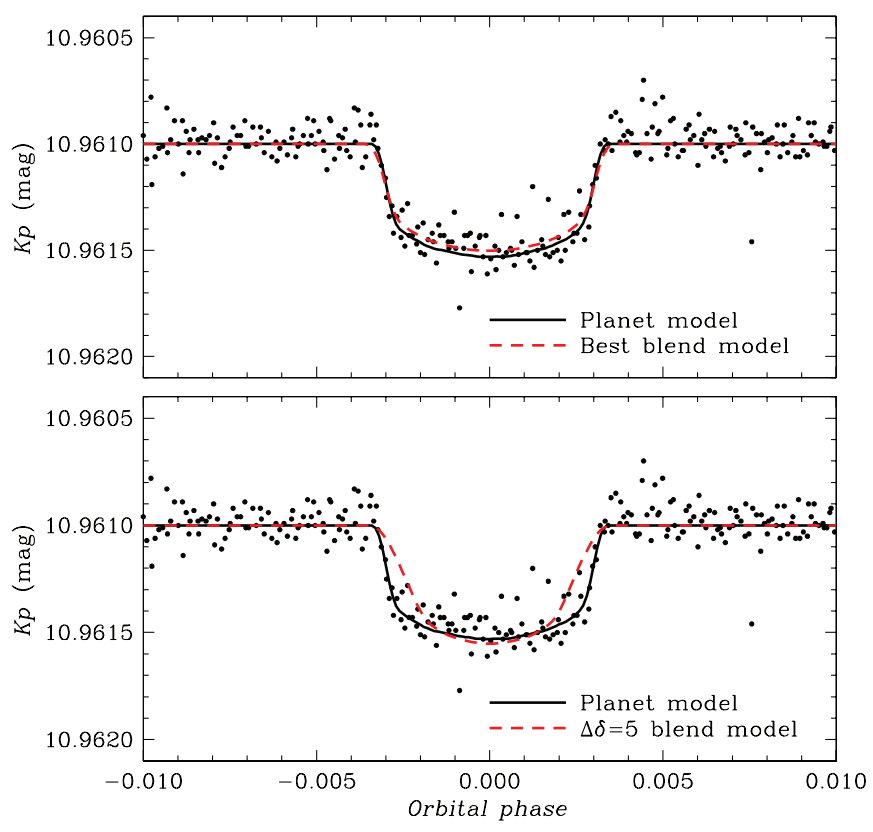

Figure 3. Long-cadence Kepler observations for KOI-072.02 compared with two different blend models involving background eclipsing binaries (red lines), and shown against the best-fit planet model for reference (black line). All models are integrated over the duration of one cadence (29.4 minutes). Top: the blend model shown is the one giving the best fit for this type of scenario ( $2.1 \sigma$ difference compared to the planet fit). Bottom: example of a blend model (indicated with a cross in Figure 2) that illustrates the use of shape information by BLENDER in a case that would naively be expected to be a viable false positive scenario (see the text). This particular scenario corresponds to a secondary mass of $M_{2}=1.0 M_{\odot}$ and a distance modulus difference of 5 mag relative to the target, giving a brightness difference in the $K p$ band of 5.6 mag. Although it matches the depth and total duration of the transit, the ingress and egress phases are not well reproduced, so that the overall quality of the fit is poor and the blend is ruled out at more than the $10 \sigma$ level.

(A color version of this figure is available in the online journal.)

be seen in the bottom panel of Figure 3, in which we show a blend model that one would naively expect should be able to match the observations, according to the crude recipe described above. This particular blend scenario is marked with a cross in Figure 2, and corresponds to $\Delta \delta=5$ and $M_{2}=1.0 M_{\odot}$, resulting in a magnitude difference of $\Delta K p=5.6$ for the EB relative to the target. While this model does yield a good match to the measured depth, and even the total duration, it does not perform nearly as well in the ingress/egress phases, which are too long when compared against the observations. The quality of this fit relative to the best planet fit, which can also be seen in the figure, corresponds to a $10.1 \sigma$ difference, and therefore BLENDER rejects it. Thus, the reason that most blends of this class can be ruled out is ultimately the high precision of the Kepler light curves, which provides a very strong constraint on the shape of the transit light curve, and in particular on the size ratio between the secondary and tertiary, which sets the duration of the ingress and egress phases.

\subsubsection{Background/Foreground Star+Planet Pairs}

There is a very broad range of blends consisting of a background or foreground star transited by a planet (as opposed to a star) that are found by BLENDER to give satisfactory fits to the data, as shown in Figure 4. These viable blends occupy the area below the $3 \sigma$ contour represented with a thick white line. Secondary stars of all spectral types (masses) are permitted, in principle, although in practice other constraints described 


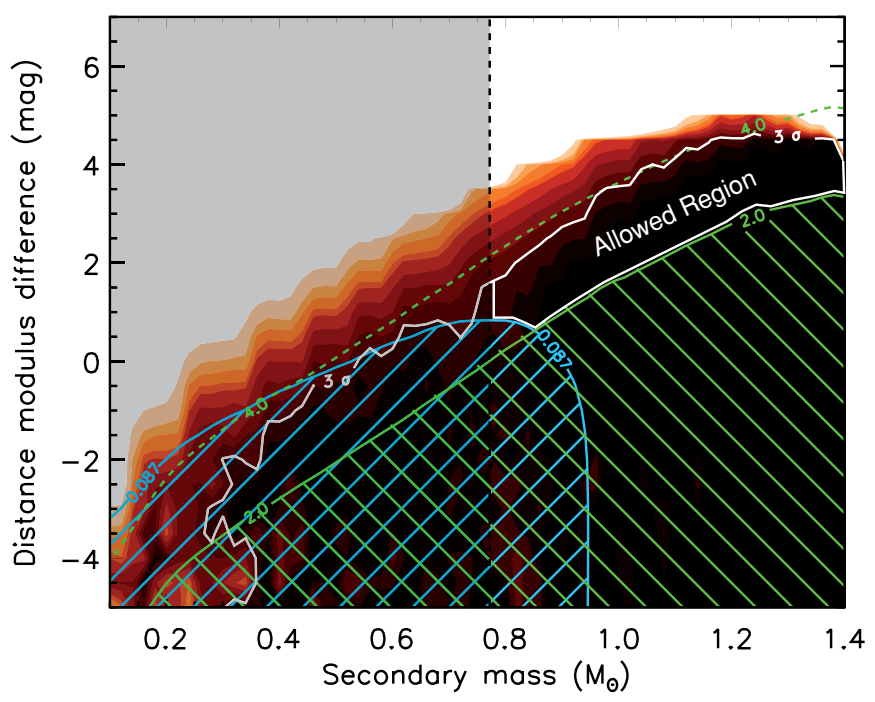

Figure 4. Similar to Figure 2, for blends involving background systems consisting of a star transited by a planet. The color scheme is the same as in Figure 2. Blends giving fits no worse than $3 \sigma$ from the best planet fit are below the thick white contour labeled with that confidence level. The shaded left-hand side of the diagram corresponds to secondary masses excluded by constraints from the Spitzer observations. Lines of constant magnitude difference relative to the target are shown in green, running diagonally from the lower left to the upper right. The dashed one at the top represents the boundary for the faintest viable blends (tangent to the white $3 \sigma$ contour). The solid green line below and parallel to it $(\Delta K p=2 \mathrm{mag})$ and hatched region to the right marks the area of parameter space excluded by our spectroscopic constraints. The blue curve and hatched region to the left represent blends that are excluded because they are too red in $K p-K_{s}$ compared to the target. Note that the colors of the blended stars are computed from a different isochrone than that of the target, which explains why blends with secondaries of the same mass as the target are ruled out for being too red. The combination of all these constraints leaves only a reduced area of parameter space (labeled "Allowed Region") where blend models give tolerable fits to the Kepler light curve and are not ruled out by any of our follow-up observations.

(A color version of this figure is available in the online journal.)

below eliminate a substantial fraction of them. All of these blends involve secondary+tertiary pairs that are within 4 mag of the target in the Kepler passband (diagonal dashed line in the figure). The tertiary sizes in these blends range from $0.42 R_{\text {Jup }}$ to $1.84 R_{\text {Jup }}$.

Our Warm Spitzer observations set a lower limit of about $0.77 M_{\odot}$ for the secondary masses of these blends, as described earlier; scenarios involving redder stars would result in transits at $4.5 \mu \mathrm{m}$ significantly deeper than we observe (i.e., deeper than the measured depth $+3 \sigma)$. This exclusion region is indicated by the shaded area. Additionally, blends that are much brighter than $\Delta K p=2$ would most likely have been detected spectroscopically (see Batalha et al. 2011), so we consider those to be ruled out as well. We indicate this with the green hatched region in the lower right-hand side of the figure. Finally, the colors of the background/foreground configurations simulated with BLENDER provide a further constraint which is represented by the blue hatched area on the lower left of the figure. This swath of parameter space is excluded because the blends are significantly redder than the color index measured for Kepler-10 $\left(K p-K_{s}=1.465 \pm 0.029\right)$, by more than three times the uncertainty in the observed index. As a result of these complementary constraints, the only section of parameter space remaining for viable blends involving star+planet pairs is the area under the $3 \sigma$ contour and limited from below and on the left by the hatched areas (color and brightness conditions) and shaded area (Spitzer constraint), respectively. All of these blends

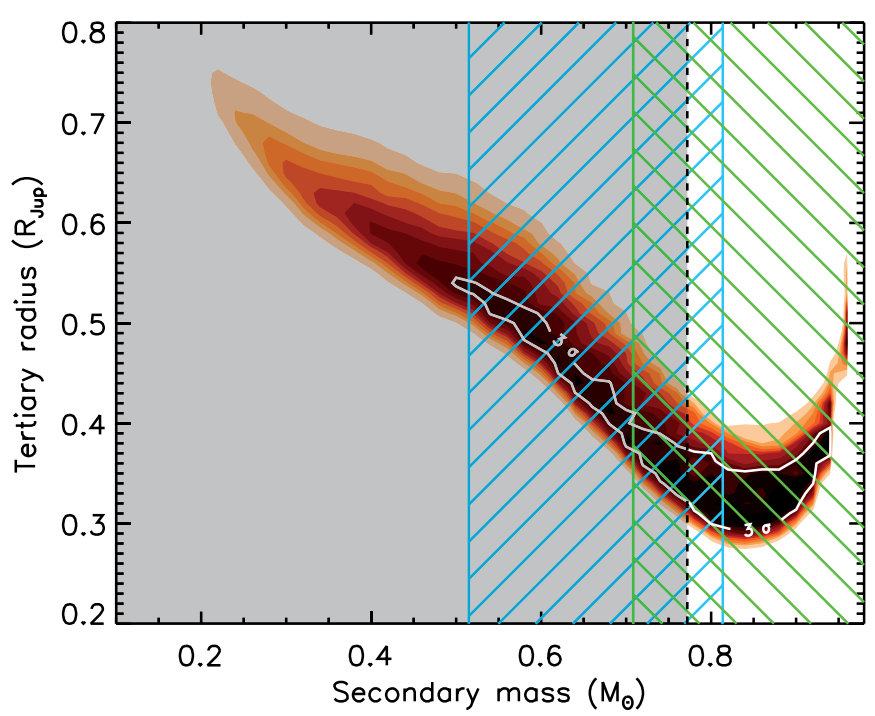

Figure 5. Similar to Figure 2, for the case of hierarchical triple systems in which the secondary star is transited by a planet. The color scheme is the same as in Figure 2. In this case the vertical axis shows the tertiary sizes. The constraints from Spitzer (gray shaded area to the left of $0.77 M_{\odot}$ ), color information (blue hatched area on the left), and spectroscopy (green hatched area on the right) are shown as in previous figures. When taken together these constraints eliminate all blends of this kind.

(A color version of this figure is available in the online journal.)

have the eclipsing pair behind the target (foreground scenarios are all ruled out).

We note that in this star+planet blend scenario, white dwarfs can also act as tertiaries as long as they are cooler than the secondaries so that they do not lead to deep occultation events that would have been seen in the light curve of KOI-072.02. The above range of tertiary radii $\left(0.42 R_{\text {Jup }}\right.$ to $\left.1.84 R_{\text {Jup }}\right)$ excludes essentially all cool carbon-oxygen and oxygen-neon white dwarfs more massive than about $0.4 M_{\odot}$, as these are smaller than the lower limit set by BLENDER, which corresponds to $4.7 R_{\oplus}$ (see, e.g., Panei et al. 2000). Low-mass helium-core or oxygen-core white dwarfs that are the product of commonenvelope evolution in binary stars can be considerably larger in size, although they appear to be very rare. The Kepler mission itself has uncovered only three examples to date (Rowe et al. 2010; Carter et al. 2011); however, all of them are very hot $\left(T_{\text {eff }}>10,000 \mathrm{~K}\right)$ and produce deep and unmistakable flatbottomed occultation signals. Model calculations such as those of Panei et al. (2007) show that as these helium-core white dwarfs cool, their radii quickly become Earth-size or smaller. Therefore, we do not consider white dwarfs to be a significant source of blends for KOI-072.02.

\subsubsection{Hierarchical Triple Scenarios (Star+Star and Star+Planet Blends)}

Eclipsing binaries composed of two stars physically associated with the target are clearly ruled out by BLENDER, as they produce very poor fits to the Kepler light curves. For cases in which the tertiaries are planets, viable scenarios identified by BLENDER span a range of secondary masses and tertiary radii within the $3 \sigma$ contour shown in Figure 5. Most of these configurations turn out to involve eccentric orbits, with transit durations longer than those corresponding to circular orbits along with secondary stars that are smaller than the primary (see Figure 6). Once again other observational constraints are very complementary, and in this case they are sufficient to exclude 


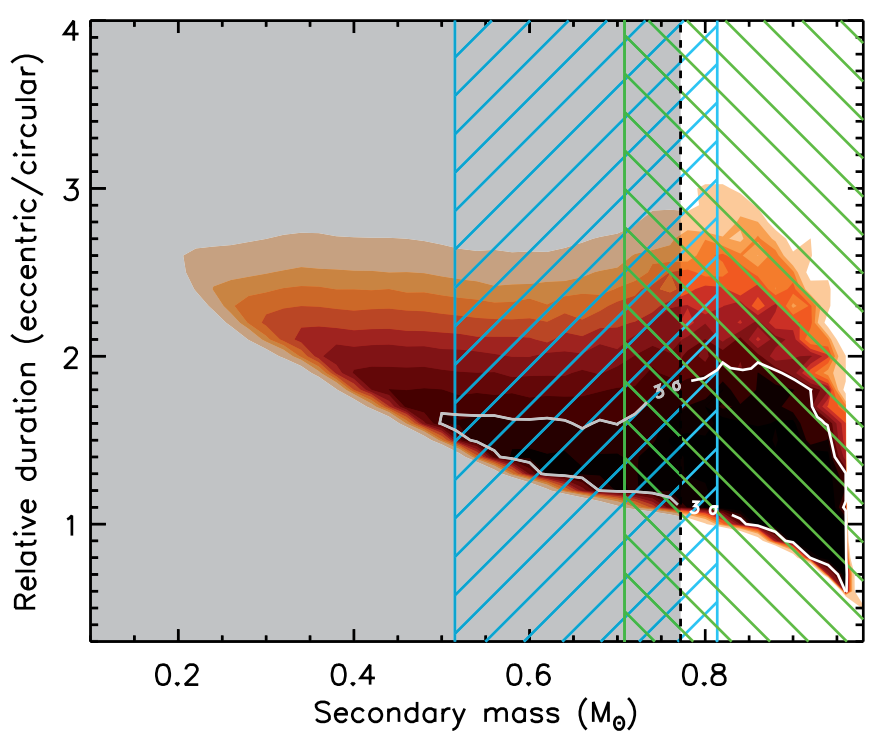

Figure 6. Same as Figure 5, but with the vertical axis showing the relative transit durations $\left(D / D_{\text {circ }}\right)$.

(A color version of this figure is available in the online journal.)

all of these blends. For example, the shaded area of parameter space to the left of $0.77 M_{\odot}$ is eliminated by the Spitzer observations, as described earlier. The constraint on the $K p-K_{s}$ color (hatched area on the left) is partly redundant with the NIR observations, but extends to slightly larger secondary masses. And finally, the spectroscopic constraint removes the remaining scenarios corresponding to higher-mass (brighter) secondaries.

We conclude that of all the hierarchical triple blend scenarios that are capable of precisely reproducing the detailed shape of the Kepler transit light curve, none would have escaped detection by one or more of our follow-up efforts, including NIR Spitzer observations, high-resolution spectroscopy, or absolute photometry (colors). ${ }^{22}$ This highlights the importance of these types of constraints for validating Kepler candidates, given that blends involving physically associated stars would generally be spatially unresolved by our high-resolution imaging with $\mathrm{AO}$ or speckle interferometry, and they would typically also be below the sensitivity limits of our centroid motion analysis, so that they would not be detected by those means. Therefore, the only blends we need to be concerned about for KOI-072.02 are those consisting of stars in the background of the target that are orbited by other stars or by transiting planets.

\section{A PRIORI LIKELIHOOD OF REMAINING BLEND SCENARIOS FOR KOI-72.02}

In order to estimate the frequency of the blend scenarios (i.e., background configurations) that remain possible after applying BLENDER and all other observational constraints, we follow a procedure similar to that described by Torres et al. (2011) for Kepler-9 d. We appeal to the Besançon Galactic structure models of Robin et al. (2003) to predict the number density of background stars of each spectral type (mass) and brightness

\footnotetext{
22 The possibility, remote as it may be, that the target has a physically associated companion that is nearly of the same brightness and that has managed to elude detection is always present (see Section 3.3), not only here but in all previously discovered transiting planets. For the present purposes we do not consider this "twin star" scenario as a false positive in the strict sense (see also Torres et al. 2011), as the transiting object would still be a planet, only that it would be larger than we thought by about a factor of $\sqrt{2}$ because of the extra dilution from the companion.
}

around Kepler-10, in half-magnitude bins, and we make use of estimates of the frequencies of transiting planets and of eclipsing binaries from recent studies by the Kepler team to infer the number density of blends. Using constraints from our highresolution imaging (specifically, the sensitivity curves presented by Batalha et al. 2011, their Figure 9) we calculate the area around the target within which blends would go undetected, and with this the expected number of blends. ${ }^{23}$

The recent release by Borucki et al. (2011b) of a list of 1235 candidate transiting planets (KOIs) from Kepler provides a means to estimate planet frequencies needed for our calculations, with significant advantages over the calculations of Torres et al. (2011) for Kepler-9 d, which were based on the earlier list of candidates published by Borucki et al. (2011a). The sample is now not only much larger, but also the knowledge of the rate of false positives for Kepler is much improved, and that rate is believed to be relatively small $(20 \%-40 \%$ depending on the level of vetting of the candidate, according to Borucki et al. 2011a; less than $10 \%$ according to the recent study by Morton \& Johnson 2011). Thus, our results will not be significantly affected by the assumption that all of the candidates are planets (see also below). An additional assumption we make is that this census is largely complete. Among these candidates we count a total of 267 having radii in the range allowed by BLENDER for the tertiaries of viable blends (i.e., between 0.42 and $\left.1.84 R_{\mathrm{Jup}}\right)$. With the total number of Kepler targets being 156,453 (Borucki et al. 2011b), the relevant frequency of transiting planets for our blend calculation is $f_{\text {planet }}=267 / 156,453=0.0017$. Slawson et al. (2011) have recently published a catalog of the 2165 eclipsing binaries found in the Kepler field, from the first four months of observation. Only the 1225 detached systems among these are considered here, since binaries in the category of semi-detached, overcontact, or ellipsoidal variables would not produce light curves with a shape consistent with a transit. The frequency of eclipsing binaries for our purposes is then $f_{\mathrm{EB}}=1225 / 156,453=0.0078$.

Table 1 presents the results of our calculation of the frequency of blends, separately for background blends with stellar tertiaries (eclipsing binaries) and with planetary tertiaries. Columns 1 and 2 give the $K p$ magnitude range of each bin and the magnitude difference $\Delta K p$ relative to the target, calculated at the upper edge of each bin. Column 3 reports the mean number density of stars per square degree obtained from the Besançon models, for stars in the mass range allowed by BLENDER as shown in Figure 2. In Column 4, we list the maximum angular separation $\rho_{\max }$ at which stars in the corresponding magnitude bin would go undetected in our imaging observations, taken from the information in the work of Batalha et al. (2011). The product of the area implied by this radius and the stellar densities in the previous column give the number of stars in the appropriate mass range, listed in Column 6 in units of $10^{-6}$. Multiplying these figures by the frequency of eclipsing binaries $f_{\mathrm{EB}}$ then gives the number of background star+star blends in Column 7. A similar calculation for the background star+planet blends, making use of $f_{\text {planet }}$, is presented in Columns 7-10. We sum up the contributions from each magnitude bin at the bottom of Columns 6 and 10. The total number of blends we expect a priori $(\mathrm{BF})$ is given in the last line of the table by adding these two values together, and is $\mathrm{BF}=1.62 \times 10^{-8}$. The calculations show that background blends consisting of

\footnotetext{
23 In the case of KOI-072.02 the exclusion radius from the centroid motion analysis, $2^{\prime \prime}$ (Section 3.3), is significantly less constraining than the high-resolution imaging, so is not as useful here as it was for Kepler-9 d.
} 
Table 1

Blend Frequency Estimate for KOI-072.02

\begin{tabular}{|c|c|c|c|c|c|c|c|c|c|}
\hline \multirow[b]{2}{*}{$\begin{array}{l}K p \text { Range } \\
(\mathrm{mag})\end{array}$} & \multirow[b]{2}{*}{$\begin{array}{c}\Delta K p \\
(\mathrm{mag})\end{array}$} & \multicolumn{4}{|c|}{ Blends Involving Stellar Tertiaries } & \multicolumn{4}{|c|}{ Blends Involving Planetary Tertiaries } \\
\hline & & $\begin{array}{l}\text { Stellar Density } \\
\text { (per sq. deg) }\end{array}$ & $\begin{array}{c}\rho_{\max } \\
\left({ }^{\prime \prime}\right) \\
(4)\end{array}$ & $\begin{array}{c}\text { Stars } \\
\left(\times 10^{-6}\right) \\
(5)\end{array}$ & $\begin{array}{c}\text { EBs } \\
f_{\mathrm{EB}}=0.78 \% \\
\left(\times 10^{-6}\right) \\
(6)\end{array}$ & $\begin{array}{l}\text { Stellar Density } \\
\quad \text { (per sq. deg) }\end{array}$ & $\begin{array}{c}\rho_{\max } \\
\left({ }^{\prime \prime}\right) \\
(8)\end{array}$ & $\begin{array}{c}\text { Stars } \\
\left(\times 10^{-6}\right) \\
(9)\end{array}$ & $\begin{array}{c}\text { Transiting Planets } \\
0.42-1.84 R_{\text {Jup }}, f_{\text {Plan }}=0.17 \% \\
\left(\times 10^{-6}\right) \\
(10)\end{array}$ \\
\hline $11.0-11.5$ & 0.5 & $\ldots$ & $\ldots$ & $\ldots$ & $\ldots$ & $\ldots$ & $\ldots$ & $\ldots$ & $\ldots$ \\
\hline $11.5-12.0$ & 1.0 & $\ldots$ & $\ldots$ & $\ldots$ & $\ldots$ & $\ldots$ & $\ldots$ & $\ldots$ & $\ldots$ \\
\hline $12.0-13.0$ & 1.5 & $\ldots$ & $\ldots$ & $\ldots$ & $\ldots$ & $\cdots$ & $\ldots$ & $\ldots$ & $\ldots$ \\
\hline $12.5-13.0$ & 2.0 & $\ldots$ & $\ldots$ & $\ldots$ & $\ldots$ & $\ldots$ & $\ldots$ & $\ldots$ & $\ldots$ \\
\hline $13.0-13.5$ & 2.5 & $\ldots$ & $\ldots$ & $\ldots$ & $\ldots$ & 139 & 0.12 & 0.485 & 0.0008 \\
\hline $13.5-14.0$ & 3.0 & 32 & 0.15 & 0.175 & 0.0014 & 197 & 0.15 & 1.074 & 0.0018 \\
\hline $14.0-14.5$ & 3.5 & 44 & 0.18 & 0.346 & 0.0027 & 278 & 0.18 & 2.183 & 0.0037 \\
\hline $14.5-15.0$ & 4.0 & $\ldots$ & $\ldots$ & $\ldots$ & $\ldots$ & 351 & 0.20 & 3.403 & 0.0058 \\
\hline $15.0-15.5$ & 4.5 & $\ldots$ & $\ldots$ & $\ldots$ & $\ldots$ & $\ldots$ & $\ldots$ & $\ldots$ & $\ldots$ \\
\hline $15.5-16.0$ & 5.0 & $\ldots$ & $\ldots$ & $\ldots$ & $\ldots$ & $\ldots$ & $\ldots$ & $\ldots$ & $\ldots$ \\
\hline $16.0-16.5$ & 5.5 & $\ldots$ & $\ldots$ & $\ldots$ & $\ldots$ & $\ldots$ & $\ldots$ & $\ldots$ & $\ldots$ \\
\hline $16.5-17.0$ & 6.0 & $\ldots$ & $\ldots$ & $\ldots$ & $\ldots$ & $\ldots$ & $\ldots$ & $\ldots$ & $\ldots$ \\
\hline $17.0-17.5$ & 6.5 & $\ldots$ & $\ldots$ & $\ldots$ & $\ldots$ & $\ldots$ & $\ldots$ & $\ldots$ & $\ldots$ \\
\hline $17.5-18.0$ & 7.0 & $\ldots$ & $\ldots$ & $\ldots$ & $\ldots$ & $\ldots$ & $\ldots$ & $\ldots$ & $\ldots$ \\
\hline $18.0-18.5$ & 7.5 & $\ldots$ & $\ldots$ & $\ldots$ & $\ldots$ & $\ldots$ & $\ldots$ & $\ldots$ & $\ldots$ \\
\hline $18.5-19.0$ & 8.0 & $\ldots$ & $\ldots$ & $\ldots$ & $\ldots$ & $\ldots$ & $\ldots$ & $\ldots$ & $\ldots$ \\
\hline \multicolumn{2}{|l|}{ Totals } & 76 & $\ldots$ & 0.521 & 0.0041 & 965 & $\ldots$ & 7.145 & 0.0121 \\
\hline & & & & frequenc & $F)=(0.0041$ & 1) $\times 10^{-6}=1$ & $10^{-8}$ & & \\
\hline
\end{tabular}

Notes. Magnitude bins with no entries correspond to brightness ranges in which all blends are ruled out by a combination of BLENDER and other constraints.

a The number densities in Columns 3 and 7 differ because of the different secondary mass ranges permitted by BLENDER for the two kinds of blend scenarios, as shown in Figures 2 and 4.

star+planet pairs contribute to this frequency about three times more than background eclipsing binaries.

While we have assumed up to now that any companions to KOI-072.02 within $\Delta K p=2$ mag of the target would have been seen spectroscopically, we note that relaxing this condition to a much more conservative $\Delta K p=1$ has no effect at all on the contribution from eclipsing binaries, and a negligible effect on the contribution of star+planet scenarios.

\section{LIKELIHOOD OF THE PLANET INTERPRETATION FOR KOI-72.02}

To obtain a Bayesian estimate of the probability that KOI-072.02 is indeed a planet as opposed to a false positive (or equivalently, the "FAR") we follow the general methodology of Torres et al. (2011) and compare the a priori likelihoods of blends and of planets: $F A R=B F / P F$. If the a priori $\mathrm{BF}$ is sufficiently small compared the planet frequency $(\mathrm{PF})$, we consider the planet validated. Our a priori blend frequencies above correspond to false positive scenarios giving fits to the light curve that are within $3 \sigma$ of the best planet fit. We use a similar criterion to estimate the a priori PF by counting the KOIs in the Borucki et al. (2011b) sample that have radii within $3 \sigma$ of the best fit from a planet model ( $R_{p}=2.227_{-0.057}^{+0.052} R_{\oplus}$; see Table 2 below). We find that 157 among the $1235 \mathrm{KOIs}$ are in this radius range $\left(2.06-2.38 R_{\oplus}\right)$, giving $\mathrm{PF}=157 / 156,453=0.0010$. This results in a FAR for KOI-072.02 of FAR $=1.6 \times 10^{-5}$, which is so small that it allows us to validate the candidate with a very high level of confidence. The planet is designated Kepler-10 c.

This result rests heavily on the a priori frequency of planets from the Kepler mission, derived from the assumption that all 1235 candidates reported by Borucki et al. (2011b) are indeed planets rather than false positives. If we were to be as pessimistic as to assume that as many as $90 \%$ of the small-size candidates are actually false positives (a similar rate of false positives as is typically found in ground-based surveys for transiting planets), and at the same time that all of the larger-size candidates that come into the BF calculation are real planets (thereby maximizing $\mathrm{BF}$ and minimizing $\mathrm{PF}$ ), the FAR would be 10 times larger than before, or $1.6 \times 10^{-4}$. This is still a very small number, and our conclusion regarding validation is unchanged. We note that a rate of false positives as high as $90 \%$ yields a PF that is strongly inconsistent not only with the expectations of Borucki et al. (2011b) and Morton \& Johnson (2011), but also with the independent results of ground-based Doppler surveys as reported by Howard et al. (2010).

In the above calculations we have implicitly assumed similar period distributions for planets of all sizes and for eclipsing binaries. However, it is conceivable that the results could change if the period distribution of planets such as Kepler-10 c were significantly different from the one for larger planets that go into the BF calculations, or from the one for EBs (which have a smaller contribution to BF; see Table 1). Therefore, as a further test, we considered the impact of restricting the periods to be within an arbitrary factor of two of the Kepler-10 c period of 45.3 days, both in our BF calculations and for the a priori estimate of the PF. We find that the planet frequencies are reduced by a factor of 4.5 , and the EB frequency by a factor of 10.4, and as a result the FAR for KOI-072.02 is FAR $=1.4 \times$ $10^{-5}$, which is about the same as before. Thus, our conclusions are robust against assumptions about the period distributions.

Finally, our FAR is conservative in the sense that we have not accounted for the flatness (coplanarity) of the Kepler-10 system. Only a small fraction of single transiting planets with periods as long as 45 days orbiting background stars (i.e., those acting as blends) are likely to transit, a priori, whereas a planet of this period such as Kepler-10 c is much more likely to transit if it is 
Table 2

Star and Planet Parameters for Kepler-10 c

\begin{tabular}{|c|c|c|}
\hline Parameter & Value & Notes \\
\hline \multicolumn{3}{|c|}{ Spectroscopically determined stellar parameters } \\
\hline Effective temperature, $T_{\text {eff }}(\mathrm{K})$ & $5627 \pm 44$ & A \\
\hline Surface gravity, $\log g(\mathrm{cgs})$ & $4.35 \pm 0.06$ & A \\
\hline Metallicity, $[\mathrm{Fe} / \mathrm{H}]$ & $-0.15 \pm 0.04$ & A \\
\hline Projected rotation, $v \sin i\left(\mathrm{~km} \mathrm{~s}^{-1}\right)$ & $0.5 \pm 0.5$ & A \\
\hline \multicolumn{3}{|l|}{ Inferred host star properties } \\
\hline Mass, $M_{\star}\left(M_{\odot}\right)$ & $0.895 \pm 0.060$ & B \\
\hline Radius, $R_{\star}\left(R_{\odot}\right)$ & $1.056 \pm 0.021$ & $\mathrm{~B}$ \\
\hline Surface gravity, $\log g_{\star}(\mathrm{cgs})$ & $4.341 \pm 0.012$ & B \\
\hline Luminosity, $L_{\star}\left(L_{\odot}\right)$ & $1.004 \pm 0.059$ & B \\
\hline Absolute $V$ magnitude, $M_{V}$ (mag) & $4.746 \pm 0.063$ & $\mathrm{~B}$ \\
\hline Age (Gyr) & $11.9 \pm 4.5$ & B \\
\hline Distance $(\mathrm{pc})$ & $173 \pm 27$ & B \\
\hline \multicolumn{3}{|l|}{ Transit and orbital parameters } \\
\hline Orbital period, $P$ (days) & $42.29485_{-0.00076}^{+0.00065}$ & $\mathrm{C}$ \\
\hline Mid-transit time, $T_{c}$ (HJD) & $2,454,971.6761_{-0.0023}^{+0.0020}$ & $\mathrm{C}$ \\
\hline Scaled semimajor axis, $a / R_{\star}$ & $49.1_{-1.3}^{+1.2}$ & $\mathrm{C}$ \\
\hline Scaled planet radius, $R_{\mathrm{p}} / R_{\star}$ & $0.01938_{-0.00024}^{+0.00020}$ & $\mathrm{C}$ \\
\hline Impact parameter, $b$ & $0.299_{-0.073}^{+0.089}$ & $\mathrm{C}$ \\
\hline Orbital inclination, $i$ (deg) & $89.65_{-0.12}^{+0.09}$ & $\mathrm{C}$ \\
\hline Transit duration, $\Delta(\mathrm{hr})$ & $6.863_{-0.068}^{+0.065}$ & $\mathrm{C}$ \\
\hline \multicolumn{3}{|l|}{ Parameters for Kepler-10 c } \\
\hline Radius, $R_{\mathrm{p}}\left(R_{\oplus}\right)$ & $2.227_{-0.057}^{+0.052}$ & $\mathrm{~B}, \mathrm{C}$ \\
\hline Mass, $M_{\mathrm{p}}\left(M_{\oplus}\right)$ & $<20$ & $\mathrm{D}$ \\
\hline Mean density, $\rho_{\mathrm{p}}\left(\mathrm{g} \mathrm{cm}^{-3}\right)$ & $<10$ & $\mathrm{D}$ \\
\hline Orbital semimajor axis, $a(\mathrm{AU})$ & $0.2407_{-0.0053}^{+0.0044}$ & $\mathrm{E}$ \\
\hline Equilibrium temperature, $T_{\mathrm{eq}}(\mathrm{K})$ & 485 & $\mathrm{~F}$ \\
\hline
\end{tabular}

Notes. In most cases these parameters are taken from Batalha et al. (2011). A: based on an analysis by D. Fischer of the Keck/HIRES template spectrum using Spectroscopy Made Easy (see Valenti \& Piskunov 1996; Batalha et al. 2011); B: based on the asteroseismology analysis and stellar models; C: based on an analysis of the photometry; D: upper limit corresponding to three times the $68.3 \%$ credible interval from the MCMC mass distribution; E: based on Newton's revised version of Kepler's third law and the results from D; F: calculated assuming a Bond albedo of 0.1 and complete redistribution of heat for re-radiation.

coplanar with Kepler-10 b. Taking this into account would boost the PF and decrease the FAR by as much as an order of magnitude (see, e.g., Beatty \& Seager 2010). Coplanarity in multiple systems is in fact supported by the large number of multiple transiting system candidates found by Kepler (Borucki et al. 2011b; Latham et al. 2011), and their mutual inclinations seem to be small ( $1^{\circ}-5^{\circ}$; Lissauer et al. 2011b). Therefore, we consider our estimate of the FAR for Kepler-10 c to be conservative.

\section{DISCUSSION}

The stellar, orbital, and planetary parameters inferred for the system as determined by Batalha et al. (2011) are summarized in Table 2, to which we add the transit duration. The small formal uncertainty in the planetary radius $(\sim 2.4 \%)$ derives from the relatively high precision of the stellar radius, which is based on asteroseismic constraints on the mean density of the star. With its radius of about $2.2 R_{\oplus}$, Kepler-10 c is among the smallest exoplanets discovered to date. The mass is undetermined as the Doppler signature has not been detected. Nevertheless, Batalha et al. (2011) placed a constraint on it based on the distribution of masses resulting from the Markov Chain Monte Carlo (MCMC) fitting procedure they applied to the existing RV measurements of Kepler-10. Their conservative $3 \sigma$ upper limit for the mass is $20 M_{\oplus}$. The corresponding maximum mean density is $10 \mathrm{~g} \mathrm{~cm}^{-3}$.

Given a precise radius measurement and mass upper limit of $20 M_{\oplus}$, some minimal constraints can be placed on the composition of Kepler-10 c. Using the models of Fortney et al. (2007), we find that an Earth-like rock-iron composition is only possible at $\sim 20 M_{\oplus}$. Lower masses would require a depletion in iron compared to rock, or more likely an enrichment in low-density volatiles such as water and/or $\mathrm{H}_{2} / \mathrm{He}$ gas. A $50 / 50 \mathrm{rock} /$ water composition yields $2.23 R_{\oplus}$ at $7 M_{\oplus}$. Still lower masses are possible with an $\mathrm{H}_{2} / \mathrm{He}$ gas envelope. Using models presented in Lissauer et al. (2011a), a planet with a rock/iron core and a $5 \% \mathrm{H}_{2} / \mathrm{He}$ atmosphere (by mass) matches the measured radius of Kepler-10 c at only $3 M_{\oplus}$. A massive $20 M_{\oplus}$ core should have attained an $\mathrm{H}_{2} / \mathrm{He}$ envelope, and it would appear to be stable at Kepler-10 c's relatively modest irradiation level, which would lead to a planetary radius dramatically larger than $2.23 R_{\oplus}$. This would tend to favor a scenario where Kepler-10 c is more akin to GJ 1214b (Charbonneau et al. 2009; Bean et al. 2010; Nettelmann et al. 2010; Désert et al. 2011c) and Kepler-11 b and Kepler-11 f, which are all below $7 M_{\oplus}$ and enriched in volatiles.

The well-measured inclinations of both Kepler-10 b and Kepler-10 c allow us to put a weak constraint on the true mutual inclination $\left(\phi_{\mathrm{bc}}\right)$ between the orbital planes of the two planets. Although the relative orientation in the plane of the sky (i.e., the mutual nodal angle) is unknown, the different impact parameters and resulting apparent inclinations place a lower limit on $\phi_{\mathrm{bc}}$. As discussed by Ragozzine \& Holman (2010), the geometric limits to the mutual inclination are given by $\left|i_{b}-i_{c}\right| \leqslant \phi_{\mathrm{bc}} \leqslant i_{b}+i_{c}$, where $i_{b}=84^{\circ} 4_{-1.6}^{+1.1}$ (Batalha et al. 2011) and $i_{c}=89.65_{-0.12}^{+0.09}$ (Table 2) are the usual inclinations with respect to the line of sight. Assuming a random orientation of the lines of nodes (which does not account for the a priori knowledge that both planets are transiting), the mutual inclination is constrained to be in the interval $5.25 \leqslant \phi_{b c} \leqslant 174.05$, with the most likely values being at the extremes of this distribution. Making the reasonable supposition of non-retrograde orbits, a mutual inclination close to the lower limit of about $5^{\circ}$ is most likely for these planets. A more detailed probabilistic argument requires making assumptions about the number of planets in the Kepler-10 system.

This mutual inclination is on the high end of the distribution inferred for other Kepler multiple candidate systems $\left(1^{\circ}-5^{\circ}\right)$ by Lissauer et al. (2011b). If this mutual inclination is typical for planets in this system, then it is relatively likely (depending on the orbital period) that other planets, if present, are not transiting. When considering the set of Kepler candidates in multiple systems that have periods less than 125 days, the ratio of periods between Kepler-10 c and Kepler-10 b (which is 54.1) is by far the highest of all period ratios of neighboring pairs of Kepler candidates (the next highest being 23.4), and is even higher than the period ratios between non-neighboring planets. Clearly, there is room for multiple additional planets between Kepler-10 b and Kepler-10 c. The preponderance of tightly packed Kepler multiple candidate systems suggests that additional planets may exist, and these may be revealed in the future with more detailed transit timing variation measurements.

Kepler-10 c is the first Kepler target observed with Warm Spitzer with the aim of testing the wavelength dependence of the transit depth. This is currently the only facility available that has the capability of detecting such shallow transits at wavelengths that are sufficiently separated from the Kepler passband to be 
helpful. In this case the observations were successful, and the transit at $4.5 \mu \mathrm{m}$ is shown to have virtually the same depth as in the optical. This places a very strong constraint on the color of potential blends, which are restricted to have secondaries of similar spectral type as the primary star.

The detailed analysis of the Kepler photometry with BLENDER combined with constraints from other observations eliminates the vast majority of possible blend scenarios. This includes most background eclipsing binaries (leaving only a small range of possible spectral types and relative fluxes for the secondaries), most of the scenarios involving chance alignments with a star transited by a larger planet, and all possible hierarchical triple configurations. The latter are among the most difficult to detect observationally since they are typically spatially unresolved. The key factors that have allowed this, and made possible the validation of the planet, are the high precision of the Kepler photometry, the relatively short ingress and egress phases (which places strong constraints on the size ratio between the secondary and tertiary), and the near equatorial orientation, resulting in a relatively flat transit that leaves less freedom for the parameters of the eclipsing binaries. We expect BLENDER to be similarly effective for other Kepler candidates that show similar features in their light curves.

Kepler-10 c along with Kepler-9 d and Kepler-11 g are examples of transiting planets that have not received the usual confirmation by dynamical means that previous discoveries have enjoyed (including essentially all ground-based discoveries), in which either the Doppler signature is detected unambiguously (and verified by the lack of bisector span variations), or transit timing variations in a multiple system are directly measured (as in Kepler-9 b and c as well as the five inner planets of the Kepler-11 system). Instead, the planets in those three cases have been validated statistically, with a Bayesian approach to estimate the probability that the transit signals are due to a planet rather than a false positive. This probability has been computed by first estimating the a priori likelihood of a false positive, and then comparing it with the a priori chance of having observed a true planet. In the three cases mentioned above the ratio of the false positive to planet likelihoods is small enough that the planetary nature of the signal is established with a very high degree of confidence. For Kepler-10 c the FAR is $1.6 \times 10^{-5}$.

The recent work of Morton \& Johnson (2011) has provided a means of assessing a rough FAR for Kepler candidates as a function of the depth of the transit signal and the brightness of the object. As noted also by those authors, while these estimates are extremely valuable for statistical studies, the validation of candidates on an individual basis with a sufficiently high degree of confidence will usually require a much more detailed analysis of false positives, such as we have performed here. Masses for these objects (other than upper limits) may of course be difficult or impractical to determine in many cases, but it is worth keeping in mind that some of the most exciting candidates to be discovered by Kepler will be in this category, namely, Earthsize planets in the habitable zones of their parent stars. Except for stars of late spectral type, the RV signals will generally be very challenging to detect with the sensitivity of current instrumentation. Thus, statistical validation of planets is likely to play an important role for Kepler in the years to come.

Funding for this Discovery mission is provided by NASA's Science Mission Directorate. This research has made use of the facilities at the NASA Advanced Supercomputing Division (NASA Ames Research Center), and is based also on observations made with the Spitzer Space Telescope which is operated by the Jet Propulsion Laboratory, California Institute of Technology under a contract with NASA. Support for this work was provided by NASA through an award issued by JPL/Caltech. We thank Mukremin Kilic and Rosanne Di Stefano for helpful discussions about white dwarfs, and the anonymous referee for constructive comments.

Facilities: Kepler, Spitzer, Keck:I(HIRES), Hale, WIYN:0.9m

\section{REFERENCES}

Batalha, N. M., et al. 2011, ApJ, 729, 27

Bean, J. L., Kempton, E. M.-R., \& Homeier, D. 2010, Nature, 468, 669

Beatty, T. G., \& Seager, S. 2010, ApJ, 712, 1433

Beerer, I. M., et al. 2011, ApJ, 727, 23

Borucki, W. J., et al. 2011a, ApJ, 728, 117

Borucki, W. J., et al. 2011b, ApJ, 736, 19

Brown, T. M., Latham, D. W., Everett, M. E., \& Esquerdo, G. A. 2011, AJ, submitted (arXiv:1102.0342)

Carter, J. A., Rappaport, S., \& Fabrycky, D. 2011, ApJ, 728, 139

Charbonneau, D., et al. 2005, ApJ, 626, 523

Charbonneau, D., et al. 2009, Nature, 462, 891

Claret, A. 2000, A\&A, 363, 1081

Deming, D., et al. 2011, ApJ, 726, 95

Désert, J.-M., Lecavelier des Etangs, A., Hébrard, G., Sing, D. K., Ehrenreich, D., Ferlet, R., \& Vidal-Madjar, A. 2009, ApJ, 699, 478

Désert, J.-M., et al. 2011a, A\&A, 526, A12

Désert, J.-M., et al. 2011b, ApJ, submitted (arXiv:1102.0555)

Désert, J.-M., et al. 2011c, ApJ, 731, L40

Eastman, J., Siverd, R., \& Gaudi, B. S. 2010, PASP, 122, 935

Fazio, G. G., et al. 2004, ApJS, 154, 10

Fortney, J. J., Marley, M. S., \& Barnes, J. W. 2007, ApJ, 659, 1661

Holman, M. J., et al. 2010, Science, 330, 51

Howard, A. W., et al. 2010, Science, 330, 653

Howard, A. W., et al. 2011, arXiv:1103.2541

Howell, S. B., Everett, M. E., Sherry, W., Horch, E., \& Ciardi, D. R. 2011, AJ, 142,19

Knutson, H. A., Charbonneau, D., Allen, L. E., Burrows, A., \& Megeath, S. T. 2008, ApJ, 673, 526

Latham, D. W., et al. 2011, ApJ, 732, L24

Léger, A., et al. 2009, A\&A, 506, 287

Lissauer, J. J., et al. 2011a, Nature, 470, 53

Lissauer, J. J., et al. 2011b, ApJ, in press (arXiv:1102.0543)

Mandel, K., \& Agol, E. 2002, ApJ, 580, L171

Markwardt, C. B. 2009, in ASP Conf. Ser. 411, Astronomical Data Analysis Software and Systems XVIII, ed. D. A. Bohlender, D. Durand, \& P. Dowler (San Francisco, CA: ASP), 251

Mazeh, T. 2008, in Tidal Effects in Stars, Planets and Disks, ed. M.-J. Goupil \& J.-P. Zahn (EAS Publications Series 29; EDP Sciences), 1

Moorhead, A. V., et al. 2011, arXiv:1102.0547

Morton, T. D., \& Johnson, J. A. 2011, arXiv:1101.5630

Nettelmann, N., Fortney, J. J., Kramm, U., \& Redmer, R. 2010, ApJ, in press (arXiv:1010.0277)

Panei, J. A., Althaus, L. G., \& Benvenuto, O. G. 2000, A\&A, 353, 977

Panei, J. A., Althaus, L. G., Chen, X., \& Han, Z. 2007, MNRAS, 382, 779

Pont, F., Zucker, S., \& Queloz, D. 2006, MNRAS, 373, 231

Press, W. H., Teukolsky, S. A., Vetterling, W. T., \& Flannery, B. P. 1992, Numerical Recipes (2nd ed.; Cambridge: Cambridge Univ. Press), 650

Ragozzine, D., \& Holman, M. J. 2010, ApJ, submitted (arXiv:1006.3727)

Robin, A. C., Reylé, C., Derriére, S., \& Picaud, S. 2003, A\&A, 409, 523

Rowe, J. F., et al. 2010, ApJ, 713, L150

Sing, D. K. 2010, A\&A, 510, 21

Slawson, R. W., et al. 2011, AJ, submitted (arXiv:1103.1659)

Snellen, I. A. G., et al. 2009, A\&A, 497, 545

Torres, G., Konacki, M., Sasselov, D. D., \& Jha, S. 2004, ApJ, 614, 979

Torres, G., et al. 2011, ApJ, 727, 24

Valenti, J. A., \& Piskunov, N. 1996, A\&A, 118, 595

Werner, M. W., et al. 2004, ApJS, 154, 1

Winn, J. N. 2010, in Exoplanets, ed. S. Seager (Tucson, AZ: Univ. Arizona Press), 55 